Vol. XXIII No $3 \quad 2017$

\title{
ELECTROMAGNETIC DOSIMETRY OF RADIOFREQUENCY PORTABLE TRANSCEIVERS USED IN FRONT OF THE FACE
}

\author{
Simona MICLĂUȘ*, Ionel DUMBRAVĂ**，Viorica VOICU**, Paul BECHET* \\ *"Nicolae Bălcescu" Land Forces Academy, Sibiu, Romania \\ **National Institute for Research, Development and Testing in Electrical Engineering - \\ ICMET, Craiova, Romania \\ simo.miclaus@gmail.com, iodum@icmet.ro,programe@icmet.ro, pbechet@gmail.com
}

\begin{abstract}
Portable transceivers emitting in the UHF radiofrequency band are sources of human electromagnetic exposure, when located in front of the face. Three output power levels were tested while the exposed target was a phantom filled with dielectric liquid simulating average human head behavior at the used frequency of $446 \mathrm{MHz}$. A flat phantom and a head phantom were scanned by an automatic E-field probe robot, based on the standardized procedure, in order to report specific absorption rates of energy deposition in the head. A discussion on the obtained results is provided, together with a comparison with other similar results in the literature. Consequences upon the safe use of such devices by public or by occupational personnel in the proximity of the brain and eyes are underlined.
\end{abstract}

\section{Keywords: SAR, RF dosimetry, human exposure, near field, portable transceiver}

\section{Introduction}

The use of portable transceivers by professionals and amateur radio persons represents a situation of human exposure to non-ionizing radiation in the region of near field of radiofrequency (RF) sources. For dual-band transceivers emitting in both very high frequency (VHF) and ultra high frequency (UHF) ranges, the indicator of user's electromagnetic safety is the specific absorption rate (SAR) of energy deposition in tissues/organs/body parts [1].

When used in front of the face, such devices may produce significant electric field strength (E-field) and/or magnetic field strength (H-field) in air, incident to the head. Part of this RF energy will be deposited inside the head. Dissipation of energy will affect mainly the sensitive organs like the brain and eyes (as the most sensitive organs) by temperature increase - in the first hand. Therefore, based on thermal effects, maximum limits were imposed in safety standards for incident field levels and SAR values [1].

In order to check compliance of safe use of portable transceivers in the UHF band (which is the interest range in the present work) or to grant device certification because output powers of such devices may be as high as $5 \mathrm{~W}$ or even $8 \mathrm{~W}$, a series of performance assessments have been made by various accredited laboratories worldwide, whose results are publicly available starting from the year 2004.

Detailed and refined tests were published as technical reports - for example by Ultratech group of labs (Canada), by Celltech Labs Inc. (Canada), by Kostec Co Ltd. (Korea) or by RF Exposure Lab. (USA) [2]-[7].

Research papers on the dosimetry of such transceivers are however scarce, as 
summarized in [8]. Among the most recent and complex studies which treat the problem, but for walkie-talkies - which have lower output powers (maximum $500 \mathrm{~mW}$ ), we mention the newest approach by the group of Vermeeren [9]. Another group who analyzed the exposure level due to portable transceivers is a Polish group [10], [11]. They found that the incident Efield level for occupational exposure defined as action level by Directive 2013/35/EU [12] - was exceeded for distances up to $15 \mathrm{~cm}$ from the transceivers' antenna when the output power was $4 \mathrm{~W}$. For SAR determination in the body they used a modelling-simulation method and demonstrated that average SAR over $10 \mathrm{~g}$ of tissue may exceed Directive 2013/35/EU limits when the distance between antenna and the human body was shorter than $5 \mathrm{~cm}$.

In [13], a RF dosimetric simulation was also approached: a. the influence of arm presence in the proximity of the transceiver; b. the influence of hole body posture on the radiation pattern of the transceiver's antenna. Calculated SAR values in the human body model conducted to the conclusion that at $5 \mathrm{~W}$ output power, transceivers should be used at a distance of minimum $10 \mathrm{~cm}$ from the body in order to ensure humans' safety. Peak values of $10-\mathrm{g}$ average local SARs were calculated in various situations and found bellow $2 \mathrm{~W} / \mathrm{kg}$ from distances of $8 \mathrm{~cm}$ onward.

Analyzing the practical use of portable transceivers by professionals over a national survey focused on various occupations, in the article published in 2015 by an Israelian group [14], the authors concluded that the cumulative collective exposures over all workers within an occupation, portable transceivers exposure dominated with $96 \%$ of the total, conducting to field levels exceeding safe limits for incident field strengths and also to the highest SARs among all RF sources.

In this regard, present approach aimed at presenting a series of dosimetric results coming from tests made in an accredited laboratory with one model of dual-band portable transceiver emitting in the UHF band and with a model of walkie-talkie, both emitting on the same frequency, for comparison of the results. The target was a body phantom (model) filled with liquid owing dielectric properties of the human brain. SAR values and their attenuation in depth of the phantom were determined and analyzed, in order to identify possible situations of unsafe use.

\section{Test setup and methodology}

Two devices under test (DUT) were used for dosimetric characterization: DUT1=two-way dual-band radio transceiver UV-B5 BaoFeng Amateur Portable Radio - at two output power levels, $\mathrm{P} 1=1 \mathrm{~W} \quad(\mathrm{LOW})$ and $\mathrm{P} 2=5 \mathrm{~W}$ (HIGH); DUT2=walkie-talkie Topcom Twintalker 9100 at the output power $\mathrm{P} 3=500 \mathrm{~mW}$ (VERY LOW). The emission took place in all cases at a unique frequency $\mathrm{f}=446 \mathrm{MHz}$ (voice analog frequency modulation, channel bandwidth $=12.5 \mathrm{kHz}$, $100 \%$ duty cycle).

To simulate human head/brain situated in front of the receiver, we used two different geometrical phantoms: a specific anthropometric mannequin $($ head+torso $)=$ SAM phantom and an elliptic flat phantom=FLAT phantom, of dimensions $635 \times 435 \times 180 \mathrm{~mm}$. Both phantoms were filled with a liquid having dielectric properties similar to those of average brain at the operating frequency: relative dielectric constant $\varepsilon^{\prime}{ }_{\mathrm{r}}=43.48$ and electric conductivity $\sigma=0.87 \mathrm{~S} / \mathrm{m}$.

The methodology used for SAR measurement followed the prescriptions in standards [15], [16]. The measurement process consists in scanning the volume of the liquid filling the phantom over a selected area to find the region where the highest levels of RF energy deposition appear. At the end is reported a single value for the peak spatial-average SAR (denoted further on by SAR avg peak) over a volume that contains $1 \overline{0} \mathrm{~g}$ of tissue/liquid, in 
the shape of a cube. This value is compared against limits established in protection standards in use in Europe [1].

The measurements took place in an electromagnetically shielded room containing the equipment for RF dosimetry model SATIMO - COMOSAR controlled by OPENSAR software (Fig. 1a). An Efield probe with an omnidirectional response (triple dipole) model EP96 from SATIMO was used for scanning the liquid by immersion. The robot arm moved the probe in desired positions. The device holder of the transmitter (Fig. 1b,c) was special designed to minimize unwanted reflections and absorptions. The measurement configurations provided a range of positions-distances between the transceiver and the phantom's shell. Power drift of the transceiver should be kept not higher than $5 \%$ over the duration of continuous exposure at the maximum (a)

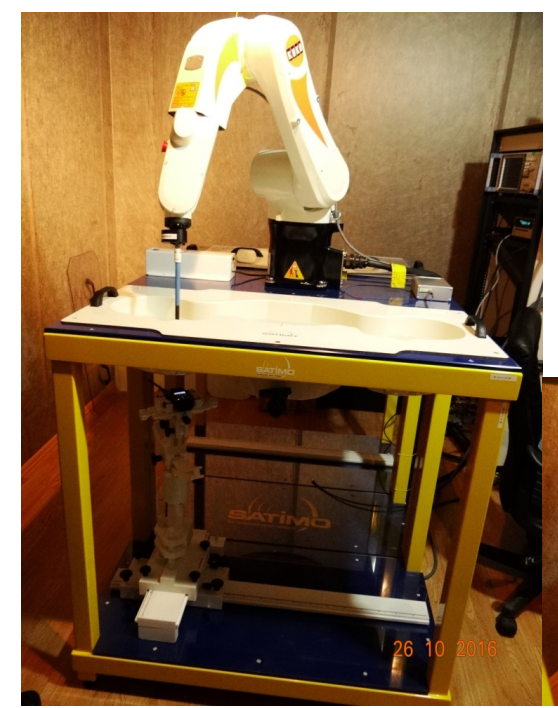

(b)

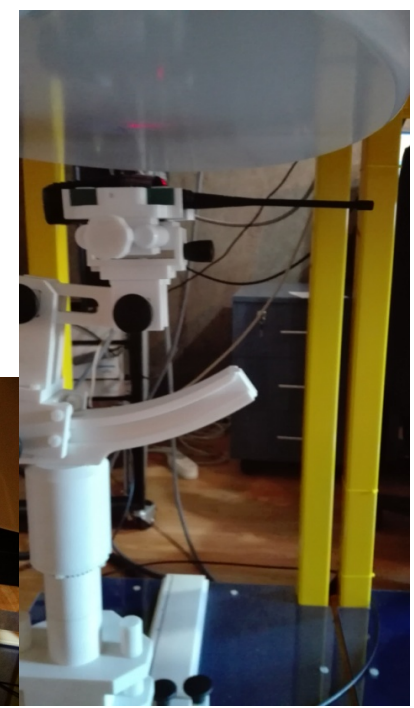

(c)

Figure 1: a. SATIMO-COMOSAR equipment used to measure SAR in the phantoms at ICMET, Craiova; $b$. positioning of the transceiver in the holder in lateral of the SAM phantom; c. positioning of the transceiver in the holder in front of the FLAT phantom.

power level, in order to minimise uncertainties. Due to the $100 \%$ duty cycle regime of emission, this condition could not be fulfilled all the time.

The measurement configuration (position) of DUTs was preserved over each single experiment. Practically, for the SAM phantom the transceiver was azimuthoriented like in Fig. 1b (parallel to the ear and the cheek), while this position was modified just in the sense of increasing the distance between the device and the SAM surface: we used D1 $=2 \mathrm{~cm}$ and $\mathrm{D} 2=7 \mathrm{~cm}$. For the FLAT phantom, DUT's surface was parallel to the planar bottom, while the distances we used were: $\mathrm{D} 1=0 \mathrm{~cm}$ ("touch position"), $\mathrm{D} 2=2 \mathrm{~cm}, \mathrm{D} 3=4 \mathrm{~cm}, \mathrm{D} 4=7 \mathrm{~cm}$,
D5 $=10 \mathrm{~cm}, \mathrm{D} 6=15 \mathrm{~cm}$.

Occupational/controlled exposure at $446 \mathrm{MHz}$ is safe if SAR averaged over whole body does not exceed $0.4 \mathrm{~W} / \mathrm{kg}$ and if SAR_avg_peak in the head and trunk doesn't exceed $10 \mathrm{~W} / \mathrm{kg}$ [1], [12]. For population, the two acceptable limits are respectively $0.08 \mathrm{~W} / \mathrm{kg}$ and $2 \mathrm{~W} / \mathrm{kg}$.

\section{Measured SAR values in the phantoms}

Near the head-held of push-to-talk devices, by using SAM and FLAT phantoms as simplified models of the head, we obtained the dosimetric results presented bellow.

Fig. 2 shows SAR attenuation in the depth of the model, when the device is distanced gradually from the phantom's shell. In Fig. 2 , on the ordinate axis is the ratio between 
local SAR value in one point of the measurement grid and the maximum SAR value reported over all scanned points in the defined grid. In Fig. 2a is represented the situation for SAM phantom with transceiver emitting in HIGH and LOW power regimes. In Fig. $2 b$ is shown the situation obtained with FLAT phantom and the transceiver in the LOW power regime, while in Fig. 2c we observe the attenuation of relative SAR for the transceiver located in front of the FLAT phantom while emitting in the HIGH power regime. Similar attenuation curves in depth of the simulated head were obtained in the case of VERY LOW power regime that is enabled with walkie-talkie. Comparing the results it is observed that the wave penetration in the flat phantom is higher in both power regimes, but not significantly. In the SAM phantom, power rate attenuation in the outer $2.5 \mathrm{~cm}$ of the model is on average of $50 \%$ from the shell value - for the HIGH POWER, and on average of $75 \%$ from the shell value - for the LOW POWER regime. Fig. 3 shows a comparison between SAR values and its distribution on a surface inside the phantoms containing the point of maximum SAR value when the emission is due to DUT2 at VERY LOW power and located $7 \mathrm{~cm}$ away from the phantom. In the FLAT phantom, SAR_avg_peak was $0.13 \mathrm{~W} / \mathrm{kg}$ while in the SAM phantom it was $0.19 \mathrm{~W} / \mathrm{kg}$. Higher local maximum SAR values were obtained with the SAM phantom than with the FLAT one, both with DUT1 and with DUT2, for the same positioning situations.

Fig. 4 emphasizes differences in SAR distribution in the SAM model when DUT1 in both power regimes is emitting at $7 \mathrm{~cm}$ distance from the head. A synthetic view of SAR_avg_peak values over all experimental cases is presented in Fig. 5, which is divided in 6 vertical zones: Zone1 and Zone3= DUT1 / LOW POWER; Zone2 and Zone4=DUT1 / HIGH POWER; Zone5 and Zone6= DUT2 / VERY LOW POWER. On the horizontal axis are presented Type of phantom / Distance $(\mathrm{cm})$. Highest SAR value corresponds to the "touch" position of the transceiver at HIGH POWER for FLAT phantom, which is $8.02 \mathrm{~W} / \mathrm{kg}$. This value doesn't exceed occupational safety limit, but it exceeds general public limit 4 times. The positioning of the transceivers in everyday
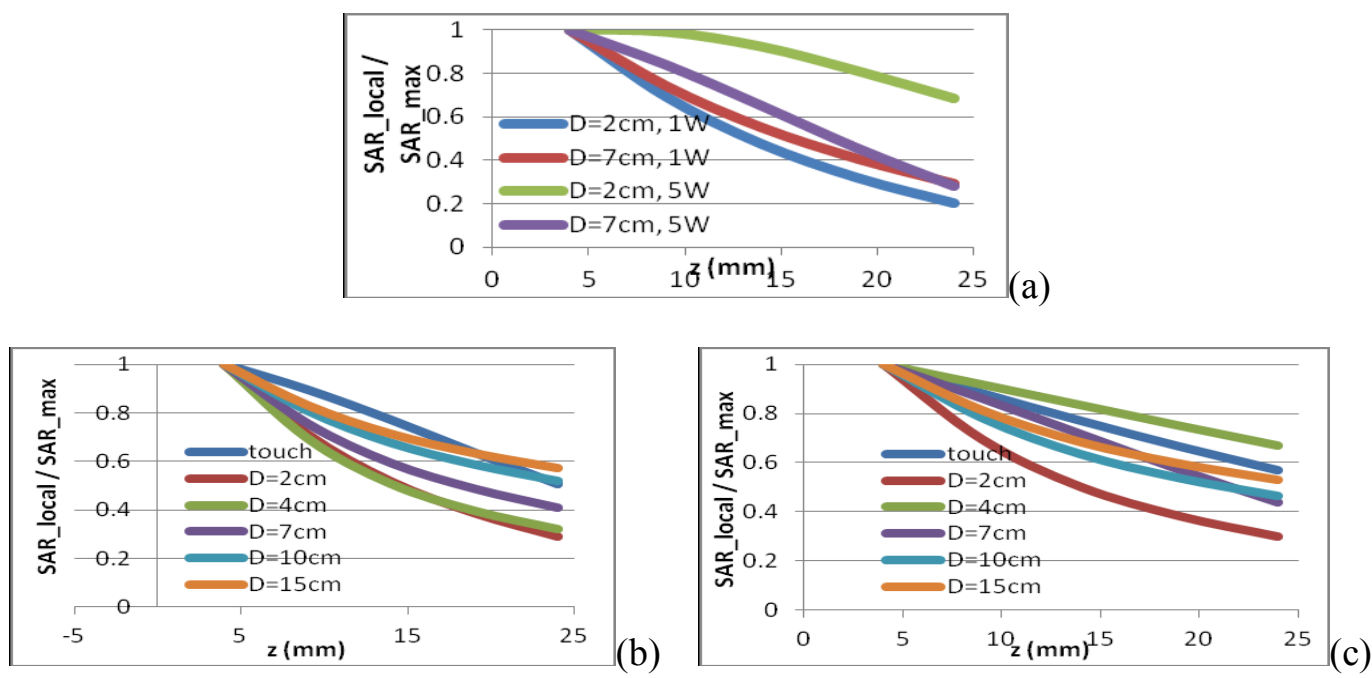

Figure 2: Attenuation of absorbed power in simulated head in phantoms - SAM (a) and FLAT (b and c), when the transceiver is held at various distances from the phantoms' surface. LOW and HIGH power emission regimes are used in (a), LOW power in (b) and HIGH power in (c) situations. 
(a)

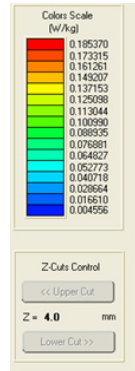

SNEN Somat

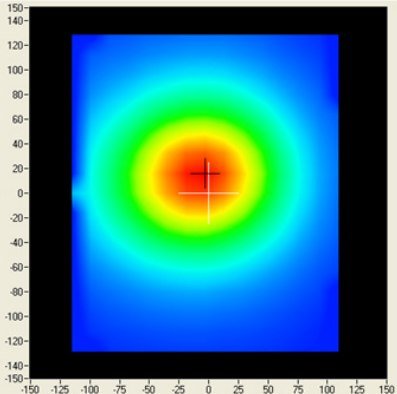

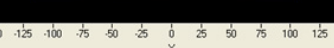

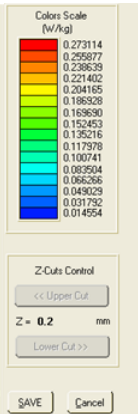

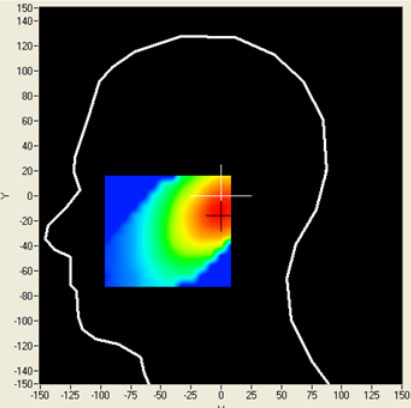

(b)

Figure 3: SAR distribution (surface radiated intensity) for walkie-talkie situated at $7 \mathrm{~cm}$ from the phantom shell: a. FLAT phantom; b. SAM phantom.

(a)

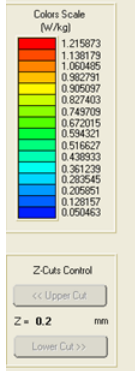

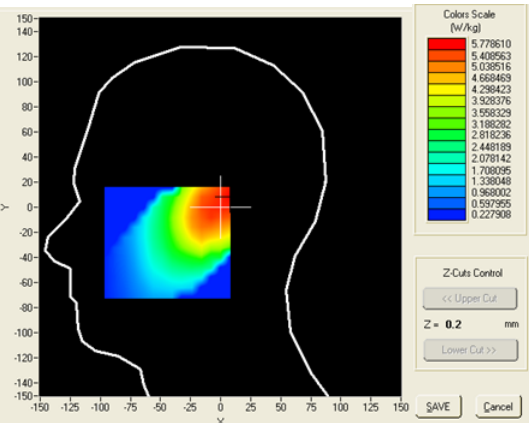

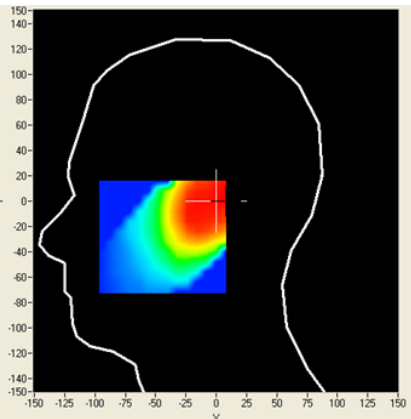

(b)

Figure 4: SAR distribution (surface radiated intensity) for transceiver placed at $7 \mathrm{~cm}$ in front of SAM phantom: a. HIGH POWER; $b$. VERY HIGH POWER.

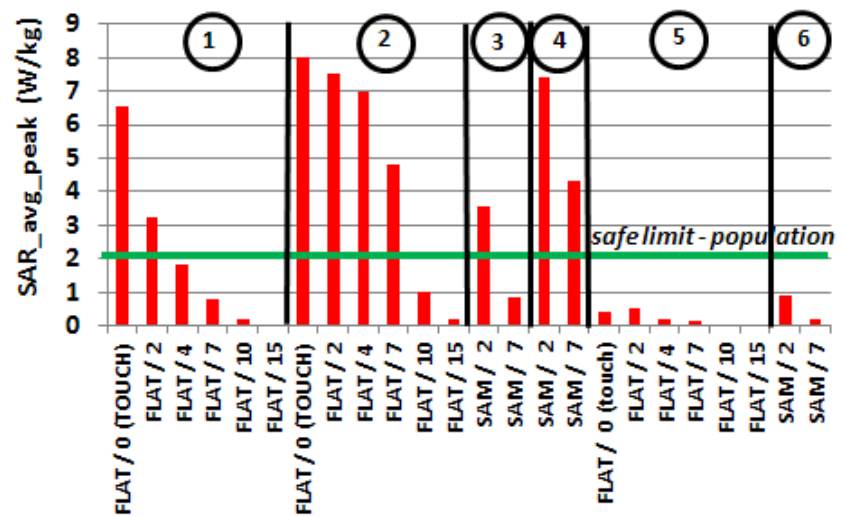

Figure 5: Average peak SAR (over 10g) values over all measured cases.

use is in the range of $7-10 \mathrm{~cm}$ from the head. When emitting in HIGH power regime, safety of population is ensured for distances larger than $8 \mathrm{~cm}$. However, occupational safety is ensured in all the situations analyzed here, because SAR_avg_peak didn't exceed $10 \mathrm{~W} / \mathrm{kg}$.

No significant differences due to type of model-phantom were observed in SAR.

\section{Conclusion}

Portable transceivers situated in front of the face, during emission at $446 \mathrm{MHz}$ and when output power is $1 \mathrm{~W}$ may exceed safety radiation limit of the pubic if used closer than $2 \mathrm{~cm}$ from the face. If the power is $5 \mathrm{~W}$ it is advised to not use the transceiver closer than $8 \mathrm{~cm}$. Walkie-talkies at $500 \mathrm{~mW}$ power are radiation-safe even when used very close to the face. However, all the discussions above were focused only on the thermal effect of RF radiation. Non-thermal effects cannot be excluded. 


\section{References}

[1] International Commission on Non-Ionizing Radiation Protection (ICNIRP), Guidelines for limiting exposure to time varying electric, magnetic, and electromagnetic fields, Health Phys., vol. 74, pp. 494-522, 1998.

[2] UltraTech Group of Labs, Engineering test report - UHF Transceiver ICOM, Model No.: IC-F24/S, UltraTech's File \#: ICOM-095-SAR, Oct. 2004.

[3] Celltech Lab. Inc., Declaration of compliance - SAR test report, Transceiever Vertex Standard Co LTD, model VX-231-AG7B-5, Aug. 2008.

[4] Celltech Lab. Inc., Declaration of compliance - SAR RF exposure evaluation, Transceiever Kenwood TK 3402U-K, Oct. 2012.

[5] UltraTech Group of Labs, Engineering test report - UHF Transceiver ICOM, Model No.: IC-F4011, UltraTech's File No.: ICOM-306Q-SAR, Dec. 2012.

[6] RF Exposure Lab., Certificate of compliance - SAR evaluation, Transceiver Midland Radio Corp. model BR200 (MMABR200), Report Number: SAR.20151008, Oct. 2015.

[7] Kostec Co. Ltd., FCC SAR test report - for UHF transceiver Sam Young Tech. Co., Ltd., model SY4050-LM, Oct. 2015.

[8] Miclaus S., Dumbrava I., Voicu V., Bechet P., Patru I., Electromagnetic Exposure due to Portable Two-way Radio Transceivers and Walkie-Talkies, Proceedings of the 10th International Symposium On Advanced Topics In Electrical Engineering, Bucharest, Romania, 23-25 March, 2017.

[9] Vermeeren G., Joseph W., and Martens L., SAR compliance assessment of PMR 446 and FRS walkie-talkies, Bioelectromagnetics, vol. 36(7), pp. 517-526, 2015.

[10] Gryz K., Zradziński P., Karpowicz J., and Leszko W., Measurement and assessment of electromagnetic fields near radiophones in line with provisions of european Directive 2013/35/EU and Polish labour law, Medycyna Pracy, vol. 64(5), pp. 671-680, 2013.

[11] Zradziński P., Leszko W., Karpowicz J., and Gryz K., Assessment of the portable radiophone users' exposure to electromagnetic fields, with use of numerical simulations and Directive 2013/35/EU requirements, Medycyna Pracy, 64(6), pp. 817-827, 2013.

[12] Directive 2013/35/EU, Minimum health and safety requirements regarding the exposure of workers to the risks arising from physical agents (electromagnetic fields), Off. J. European Union, 26 June 2013.

[13] Kogo N., Ikeda T., Antenna Performance of Push-to-Talk Transceiver in VHF and UHF Bands Considering Impedance Matching, Proceedings of 2013 URSI International Symposium on Electromagnetic Theory (EMTS), pp. 358-361, 20-24 May 2013.

[14] Hareuveny R., Kavet R., Shachar A., Margaliot M., and Kheifets L., Occupational exposures to radiofrequency fields: results of an Israeli national survey, Journal of Radiological Protection, vol. 35, pp. 429-445, 2015.

[15] International Electrotechnical Commission (IEC), Procedure to measure the specific absorption rate (SAR) for hand-held mobile wireless devices in the frequency range of $300 \mathrm{MHz}$ to $3 \mathrm{GHz}$, IEC62209, Geneva, Switzerland. 2001.

[16] International Electrotechnical Commission (IEC), Human exposure to radio frequency fields from hand-held and body-mounted wireless communication devices-human models, instrumentation, and procedures-part 2: Procedure to determine the specific absorption rate (SAR) in the head and body for $30 \mathrm{MHz}$ to $6 \mathrm{GHz}$ handheld and body mounted devices used in close proximity to the body, TC/ SC106/90/NP, Geneva, Switzerland, 2005. 